\title{
Predictive Variables for Sonographically Guided Corticosteroid Injection in Mild-to-Moderate Carpal Tunnel Syndrome
}

\author{
Seong Yun Chung, $\mathrm{MD}^{1}$, Jung Min Kwak, $\mathrm{MD}^{1}$, Seok Kang, $\mathrm{MD}^{1}$, \\ Seong-Ho Son, $\mathrm{PhD}^{2}$, Jae Do Kim, $\mathrm{MD}^{3}$, Joon Shik Yoon, $\mathrm{MD}, \mathrm{PhD}^{1}$
}

\begin{abstract}
${ }^{1}$ Department of Physical Medicine and Rehabilitation, Korea University Guro Hospital, Seoul;
${ }^{2}$ Department of Radio Technology Research, Electronics and Telecommunications Research Institute, Daejeon;

${ }^{3}$ Department of Physical Medicine and Rehabilitation, Graduate School of Korea University, Seoul, Korea
\end{abstract}

\begin{abstract}
Objective To assess the predictive variables after sonographically guided corticosteroid injection in carpal tunnel syndrome.

Methods A prospective, observational study was carried out on 25 wrists of 20 consecutive patients with carpal tunnel syndrome, confirmed by the American Association of Neuromuscular and Electrodiagnostic Medicine criteria, which includes clinical history, symptoms, and evidence of slowing of distal median nerve conduction. Visual analogue scale (VAS) and Boston Carpal Tunnel Questionnaire (BCTQ) were asked to the patients before and 4 weeks after the procedure. On a basis of VAS difference before and after the procedure, we divided the patients into two groups: more than $50 \%$ of VAS improving (good response group) and less than $50 \%$ of VAS improving (poor response group). Also, nerve conduction studies and ultrasound evaluations were performed prior to sonographically guided corticosteroid injection and at 4 weeks after the procedure. The cross-sectional area (CSA) of median nerve at maximal swelling point around wrist was measured by manual tracing using ultrasonography. With assessments mentioned above, we tried to assess predictive variables for prognosis after sonographically guided corticosteroid injection in carpal tunnel syndrome.

Results The CSA of median nerve at wrist measured before the procedure was significantly larger in good response group than in poor response group. Furthermore, the CSA of median nerve at wrist, symptom severity scale of BCTQ, motor/sensory latency and sensory amplitude were correlated with VAS improving.

Conclusion The CSA of median nerve at wrist is the strongest predictive value for sonographically guided corticosteroid injection in mild-to-moderate carpal tunnel syndrome.
\end{abstract}

Keywords Carpal tunnel syndrome, Ultrasonography, Corticosteroids, Treatment outcome

Received June 14, 2017; Accepted August 1, 2017

Corresponding author: Joon Shik Yoon

Department of Physical Medicine and Rehabilitation, Korea University Guro Hospital, 148 Gurodong-ro, Guro-gu, Seoul 08308, Korea. Tel: +82-2-26261500, Fax: +82-2-2626-1513, E-mail: rehab46@korea.ac.kr

ORCID: Seong Yun Chung (https://orcid.org/0000-0003-3414-075X); Jung Min Kwak (https://orcid.org/0000-0001-8776-7885); Seok Kang (https:// orcid.org/0000-0001-5614-4175); Seong-Ho Son (https://orcid.org/0000-0003-1343-1806); Jae Do Kim (https://orcid.org/0000-0001-6434-8186); Joon Shik Yoon (https://orcid.org/0000-0002-8985-279X).

(c) This is an open-access article distributed under the terms of the Creative Commons Attribution Non-Commercial License (http://creativecommons.org/ licenses/by-nc/4.0) which permits unrestricted noncommercial use, distribution, and reproduction in any medium, provided the original work is properly cited. Copyright @ 2018 by Korean Academy of Rehabilitation Medicine 


\section{INTRODUCTION}

Carpal tunnel syndrome (CTS) is a clinical disorder that is caused by the entrapment of the median nerve at the wrist, and is the most common form of peripheral nerve entrapment [1]. Also, CTS is a common work-related musculoskeletal disorder that results in significant healthcare costs as well as potential functional disability for the patient diagnosed with CTS. The clinical diagnosis of CTS is based on the symptoms and signs, and confirmed use of a neural electrodiagnostic testing to verify the diagnosis. However, the electrodiagnostic methods standardly utilized are known to be considered uncomfortable for patients, time consuming and expensive. Therefore, the sonographic detection of pathologic features of the median nerve appears to be a convenient alternative, which is also beneficial in providing a lowered cost per procedure. This procedure has showed promising diagnostic accuracy in previous literatures [1-4].

There are a variety of treatment methods for CTS. The selection of treatment for a patient relies on the severity, the duration of symptoms, and patient preferences [5]. If nonsurgical methods which include splinting the wrist, physical therapy, and injection of a corticosteroid are not effective in relieving symptoms for a patient, then a surgical release should be considered. However, it is noted that the dividing of the transverse carpal ligament, with open technique or endoscopic technique, will usually require an operating room with general anesthesia, a procedure which is known to slows the patient's recovery as well as have the potential for morbidity risks.

The sonographically guided corticosteroid injections have been shown to be as effective as surgery in several studies [6,7]. We observed that this technique has promising safety, fast recovery, and low morbidity rate. However, it should be noted that there are no generally accepted guidelines for which patients should be treated initially with an injection.

There are only a few published studies that address the influencing predictive factors for a corticosteroid injection in CTS [6,8-14]. Most of the studies above revealed the clinical and electrophysiological factors, but only one study announced a result in terms of sonographic factors. Meys et al. [14] revealed that a smaller cross-sectional area (CSA) of median nerve showed better outcome after corticosteroid injection. It is not clear which factors play a role in determining the results and outcome after a corticosteroid injection.

The aim of this study was to investigate which variable is predictive for prognosis after sonographically guided corticosteroid injection in CTS.

\section{MATERIALS AND METHODS}

\section{Participants}

This prospective observational study was conducted on study participants from April 2015 to March 2016 at Korea University Guro Hospital. We recruited the 25 wrists which were the focus of the study from 20 subjects diagnosed with CTS. The CTS diagnosis of the participants was confirmed by the American Association of Neuromuscular and Electrodiagnostic Medicine (AANEM) criteria, which includes a review of the patient clinical history, symptoms, and evidence of slowing of distal median nerve conduction [15].

The study exclusion criteria were as follows: (1) impossibility to obtain follow-up; (2) prior surgery or trauma at the wrist or median nerve; (3) previous splinting or corticosteroid injections for CTS; (4) clinical or electrophysiological evidence of conditions that could mimic CTS or interfere with its validation; (5) previous allergic reactions to methylprednisolone acetate or lidocaine hydrochloride; (6) diabetes mellitus, thyroid disease, chronic renal failure, or space-occupying lesions on clinical examination of the volar wrist; and (7) pregnancy.

The Institutional Review Board of Korea University Guro Hospital (No. KUGH15126-002) has approved this study, and the informed consent was obtained from all subjects and the general characteristics such as age, sex, height, weight and body mass index (BMI) of all patients were evaluated and recorded to determine the study results.

\section{Visual analogue scale}

Prior to a sonographically guided corticosteroid injection, the baseline pain score was evaluated using the visual analogue scale (VAS). When measured at 4 weeks after the procedure, the follow-up VAS score was measured. To compare the clinical efficacy, the percentage of the pain relief was thereby calculated using the following formula: 
Pain relief $(\%)=\frac{\text { (Baseline pain score-Follow-up pain score) }}{\text { Baseline pain score }} \times 100$.

We regard the pain relief of more than $50 \%$ of VAS improving as a good response to a sonographically guided corticosteroid injection.

\section{Boston Carpal Tunnel Questionnaire}

The Boston Carpal Tunnel Questionnaire (BCTQ) was completed by the patients before a sonographically guided corticosteroid injection and subsequently at 4 weeks after the procedure. The BCTQ consists of 11 questions of a measured symptom severity scale (SS) and 8 questions of a functional status scale (FS). The answers to each question are provided by a 1-5 scale, with a ' 1 ' indicating 'no symptoms' and the measurement of a ' 5 ' indicating 'severe symptoms.' The average scale of each question is represented in the survey. The symptom severity scale assesses symptoms with respect to severity, frequency, time, and type for each patient completing the survey. The functional status scale assesses the effect of CTS on daily life as noted by the participant [16].

\section{Nerve conduction studies}

All patients underwent nerve conduction studies (NCS) according to the protocol defined by the AANEM [15] before a sonographically guided corticosteroid injection, and subsequently at 4 weeks after the procedure. In the study, all testing was performed while maintaining the skin temperature at $34^{\circ} \mathrm{C}$. The sensory nerve action potentials (SNAPs) were obtained antidromically using surface electrodes placed over the third digit. The compound muscle action potentials (CMAPs) were recorded from the abductor pollicis brevis (APB) muscle, using surface electrodes in a belly-tendon montage.

The standard tests include the median sensory nerve conduction velocity between the third digit and the wrist segments, and the median distal motor latency from the wrist to the thenar eminence. When standard tests were normal, further segmental tests were performed over a short distance of $7 \mathrm{~cm}$ or comparative median/ulnar studies. The measurements performed and the cut-off or normal values used in our study were as follows: (1) median nerve distal sensory latency, upper limit of normal $3.7 \mathrm{~ms}$; (2) difference between the median and ulnar nerve distal sensory latencies, upper limit of normal 0.4 ms; (3) distal motor latency over the thenar, upper limit of normal $4.2 \mathrm{~ms}$; (4) median motor nerve conduction velocity, lower limit of normal $49 \mathrm{~m} / \mathrm{s}$; (5) median sensory nerve conduction velocity, lower limit of normal $49 \mathrm{~m} / \mathrm{s}$ [17]. The severity of electrophysiological CTS impairment was assessed according to the classification reported by Lee et al. [18] and divided into three groups as follows: 'mild', abnormal sensory and distal motor latency; 'moderate', mild plus low SNAP (6-15 $\mu \mathrm{V})$ and CMAP amplitude (2.1-4 mV); and 'severe', moderate plus very low SNAP $(<5 \mu \mathrm{V})$, CMAP amplitude $(<2.0 \mathrm{mV})$ and fibrillations, abnormal motor unit action potentials of APB in needle examination

\section{Ultrasound studies}

In the study, we used a 7- to $12-\mathrm{MHz}$ linear-array transducer to examine the affected wrist in all patients before sonographically guided corticosteroid injection, and at 4 weeks after the procedure.

A physiatrist conducted the ultrasound measurements while blinded to the electrodiagnostic findings, and was not advised of the timing of the scheduled follow-up examinations. During the analysis, the patients were maintained in the supine position, with the forearm supinated and the wrist was placed in a neutral position to prevent the appearance of the median nerve from changing according to wrist position. The transducer was first used to obtain a transverse view of the median nerve at the distal wrist crease (DWC), and moved distally and proximally in order to find the site of maximal nerve swelling. At this site, the CSA of the median nerve was measured by manual tracing of the nerve circumference, excluding the hyperechoic epineurial rim. Finally, three serial measurements were performed for averaging the results of the recorded measurements.

\section{Procedures}

During the study, all patients underwent a sonographically guided corticosteroid injection. The procedure was performed in the following manner: the patients were maintained in the sitting position, with their forearm supinated on a pillow for the maintenance of a sterile condition. The median nerve was visualized at this maximal swelling point using a 13-MHz multifrequency linear array ultrasound transducer (Philips HD15 ultrasound system and transducers). After sterilizing the skin with betadine and chlorhexidine, the injection technique was 
conducted using the ulnar side approach. The needle was inserted right proximal to the pisiform bone, approaching parallel to the flexor retinaculum, and a swollen median nerve beneath it was observed (Fig. 1). Using continuous sonographic guidance, $5 \mathrm{~mL}$ of a solution of $0.5 \%$ lidocaine and triamcinolone 1 ample $(40 \mathrm{mg} / \mathrm{mL})$ was injected over the median nerve and under the flexor retinaculum, while the remainder was injected under the median nerve. Moreover, a mechanical carpal tunnel release was attempted with the use of an inserted needle. In the study, special care had been taken to avoid any injury to vessels and nerves during the study.

After 4 weeks, the patients were asked to visit our hospital not only to see the effectiveness and any complication, but also to be evaluated by VAS, BCTQ, NCS and sonography.

\section{Statistical analysis}

The statistical analysis in this study was performed using the SPSS statistical software version 22 for Windows (IBM, Armonk, NY, USA), and the statistical significance was set at $\mathrm{p}<0.05$. The data are presented as median (interquartile range). The Wilcoxon signed-rank test was used to identify changes in variables after the study intervention. The Mann-Whitney test and chi-square test were carried out to compare the variables between the two groups for determining the applicable prognostic factors of sonographically guided corticosteroid injection in CTS. A multiple linear regression was used to evaluate the relationship of the variables to VAS improving. Also, the receiver operating characteristics (ROC) curve analysis was utilized in this case to determine the prognostic CSA value of sonographically guided corticosteroid injection in CTS.

\section{RESULTS}

Among the 25 wrists, 13 cases showed more than $50 \%$ of VAS improving after the procedure (good response group). The other 12 cases showed less than $50 \%$ of VAS improving (poor response group). There is no significant difference of baseline demographics such as age, sex, $\mathrm{BMI}$, disease duration, and electromyographic grade between the two groups of participants studied (Table 1).

All variables except the influence of the motor amplitude, showed significant improvement after the sonographically guided corticosteroid injection. In comparison with the two groups, the VAS and electrophysiological components before the procedure were not significantly different. However, the CSA of a median nerve at the wrist was measured before the procedure and showed significant difference. The symptom severity scale of BCTQ (BCTQ(SS)) completed before the procedure showed no difference. However, the BCTQ(SS) completed after the procedure showed significant differences. On the other hand, the functional severity scale of BCTQ
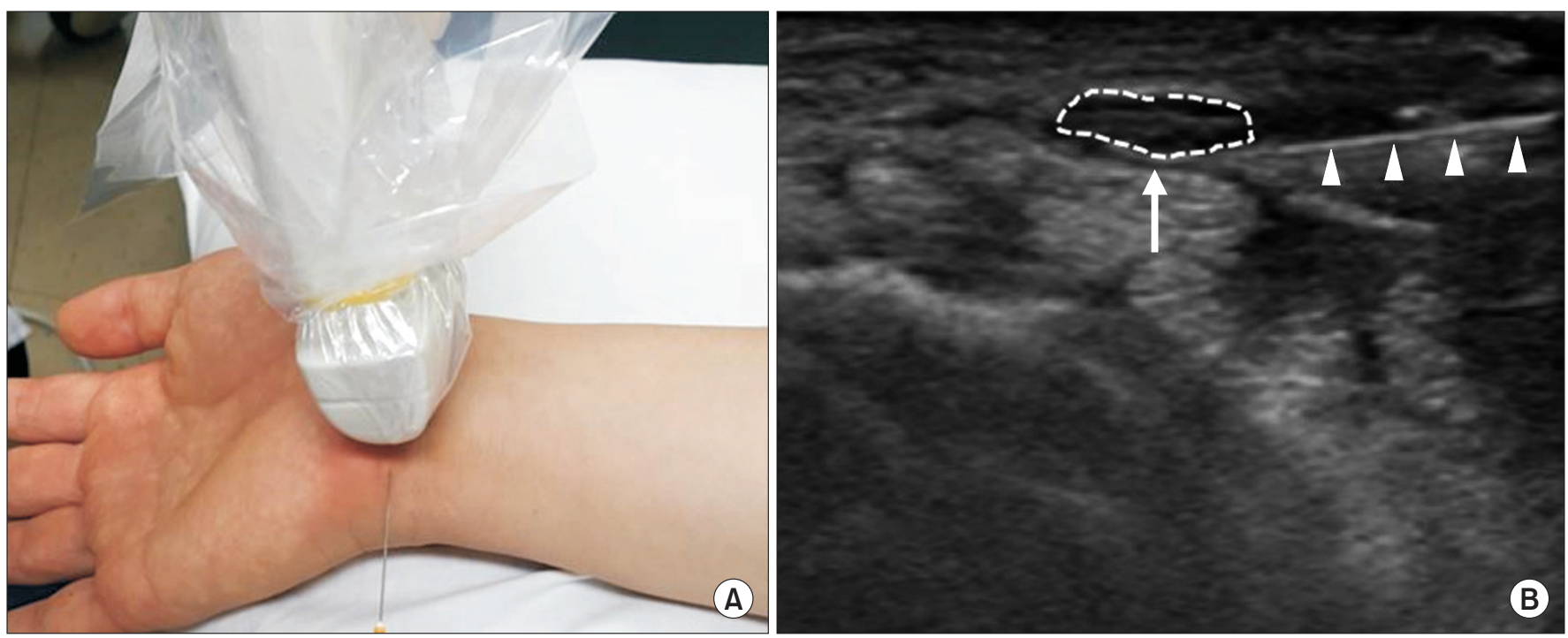

Fig. 1. Sonographically guided corticosteroid injection. (A) Needle is inserted parallel to carpal ligament. (B) Arrow with dotted line indicates median nerve; arrowheads indicate inserted needle. 
Table 1. Baseline characteristics of study subjects

\begin{tabular}{|lccc}
\hline \multicolumn{1}{|c}{ Variable } & Good response $(\mathbf{n}=\mathbf{1 3})$ & Poor response $(\mathbf{n}=\mathbf{1 2})$ & p-value \\
\hline Age $(\mathrm{yr})$ & $50.8(48.9-52.8)$ & $54.0(50.5-55.0)$ & $0.45^{\mathrm{a})}$ \\
\hline Sex & 5 & & $0.85^{\mathrm{a}}$ \\
\hline Male & 8 & 4 & \\
\hline Female & $20.1(19.8-26.5)$ & $24.1(22.9-26.6)$ & $0.15^{\mathrm{a})}$ \\
\hline BMI $\left(\mathrm{kg} / \mathrm{m}^{2}\right)$ & $12.8(12.7-13.9)$ & $9.0(2.0-21.5)$ & $0.23^{\mathrm{a})}$ \\
\hline Disease duration $(\mathrm{mo})$ & & & $0.65^{\mathrm{b})}$ \\
\hline EMG grade & 2 & 4 & \\
\hline Mild & 8 & 6 & \\
\hline Moderate & 3 & 2 & \\
\hline Severe & 3 & & \\
\hline
\end{tabular}

Values are presented as median (interquartile range).

BMI, body mass index; EMG, electromyography.

a) Mann-Whitney test.

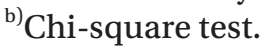

Table 2. Comparison of variables between the two groups

\begin{tabular}{|llccc}
\hline & & Good response (n=13) & Poor response (n=12) & p-value \\
\hline VAS & Pre & $6.5(5.8-7.2)$ & $6.0(5.0-6.8)$ & 0.25 \\
\hline & Post & $2.1^{* *}(1.4-2.8)$ & $3.0^{* *}(2.3-4.0)$ & $0.01^{*}$ \\
\hline BCTQ(SS) $\left(\mathrm{mm}^{2}\right)$ & Pre & $15.6(13.7-18.0)$ & $12.0(9.8-15.3)$ & $0.016^{*}$ \\
& Post & $12.5^{* *}(9.6-14.7)$ & $8.9^{* *}(8.2-14.5)$ & 0.41 \\
\hline BCTQ(FS) & Pre & $3.2(2.6-3.8)$ & $2.8(2.5-3.2)$ & 0.347 \\
\hline & Post & $1.6^{* *}(1.5-1.8)$ & $1.7^{* *}(1.6-2.3)$ & $0.04^{*}$ \\
\hline Sensory latency $(\mathrm{ms})$ & Pre & $3.4(3.2-3.6)$ & $2.2(2.0-3.0)$ & $0.001^{*}$ \\
& Post & $1.6^{* *}(1.4-1.8)$ & $1.4^{* *}(1.3-1.5)$ & 0.120 \\
\hline Sensory amplitude $(\mu \mathrm{V})$ & Pre & $4.9(4.5-9.0)$ & $4.7(4.6-5.1)$ & 0.689 \\
\hline & Post & $4.3^{* *}(3.9-4.6)$ & $4.0^{* *}(3.3-4.6)$ & 0.225 \\
\hline Motor latency $(\mathrm{ms})$ & Pre & $8.2(1.6-15.1)$ & $9.4(7.5-16.5)$ & 0.270 \\
\hline & Post & $10.7^{* *}(10.3-20.9)$ & $18.2^{* *}(13.6-32.0)$ & 0.052 \\
\hline Motor amplitude $(\mathrm{mV})$ & Pre & $4.9(4.4-5.7)$ & $5.0(4.8-5.8)$ & 0.470 \\
& Post & $4.4^{* *}(3.9-5.2)$ & $4.3^{* *}(4.0-5.3)$ & 0.894 \\
\hline
\end{tabular}

Values are presented as median (interquartile range).

VAS, visual analogue scale; CSA, cross-sectional area; BCTQ(SS), symptomatic severity scale of Boston Carpal Tunnel Questionnaire; BCTQ(FS), functional severity scale of Boston Carpal Tunnel Questionnaire.

${ }^{*} \mathrm{p}<0.05$ in Mann-Whitney test for comparison between good and poor response group.

${ }^{* *} \mathrm{p}<0.05$ in Wilcoxon signed test for comparison before and after the procedure.

(BCTQ(FS)) completed before the procedure showed significant difference. It is similarly to be noted that after the procedure, the BCTQ(FS) showed no difference (Table 2).

The result of multiple linear regression are shown in Table 3 with independent variables that had significant $(\mathrm{p}<0.05)$ correlations with VAS improving. When 9 independent variables were considered, 5 variables-CSA of median nerve at wrist, BCTQ(SS), motor latency, sensory latency and sensory amplitude-were noted as significant predictors of the improvement of VAS. To enumer- 
Table 3. Multiple linear regression coefficients for clinical efficacy

\begin{tabular}{lcc}
\hline Independent variable & Coefficient (r) & p-value \\
\hline VAS & -0.889 & 0.696 \\
CSA & 440.033 & $0.001^{*}$ \\
BCTQ(SS) & -11.322 & $0.02^{*}$ \\
BCTQ(FS) & 3.726 & 0.365 \\
\hline Sensory latency & 7.345 & $0.001^{*}$ \\
\hline Sensory amplitude & 1.970 & $0.004^{*}$ \\
\hline Motor latency & -14.318 & $0.009^{*}$ \\
\hline Motor amplitude & 0.274 & 0.585 \\
\hline Conduction velocity & -0.865 & 0.154 \\
\hline
\end{tabular}

VAS, visual analogue scale; CSA, cross-sectional area; BCTQ(SS), symptomatic severity scale of Boston Carpal Tunnel Questionnaire; BCTQ(FS), functional severity scale of Boston Carpal Tunnel Questionnaire. ${ }^{*} \mathrm{p}<0.05$.

ate, among the 5 variables, the CSA of a median nerve at the wrist showed the highest coefficient $(r=440.033)$. In other words, this result means that the CSA of a median nerve at the wrist is the most significant predictor with VAS improving after a sonographically guided corticosteroid injection.

The ROC curve analysis revealed that CSA $>1.26 \mathrm{~mm}^{2}$ (area under curve $[\mathrm{AUC}]=0.782$, sensitivity $100 \%$ and specificity $75.0 \%$ ) was the most powerful prognostic value of sonographically guided corticosteroid injection in CTS.

\section{DISCUSSION}

The result of this study indicates that the CSA of a median nerve at the wrist is the most powerful predictor for determining the outcome of a sonographically guided corticosteroid injection. Also, other measures such as BCTQ(SS), motor/sensory latency and sensory amplitude are predictive valuables as well.

The CSA of median nerve at wrist measured before the procedure was significantly larger in good response group than in poor response group. This might be explained by the fact that corticosteroid is more effective in inflammatory tissues, which is clinically shown as swollen. Moreover, the results of ROC curve show that a CSA of median nerve of $12.6 \mathrm{~mm}^{2}$ could be a cut-off value of good effect of sonographically guided corticosteroid in- jection

There are a few notable published studies that address predictive factors for utilizing a corticosteroid injection in CTS [6,8-14]. Most of the studies noted above revealed the clinical and electrophysiological factors influencing the results, but only one study announced a result in terms of sonographic factors as a trigger for the results. In the study of Meys et al. [14], the CSA of median nerve at the wrist was negatively correlated with the outcome of carpal tunnel steroid injection. However, in our study, the patients with larger CSA of median nerve showed better response to the steroid injection. In the study of Visser et al. [19], the low NCS grade of median nerve was valuable predictor of outcome after a carpal tunnel steroid injection. Namely, in our study, the results of NCS were not significantly different between the good and poor response participant groups. In those previous studies, the severities of CTS were considered severe to extreme in most of the patients studied. In cases of failure to steroid injection, the patients underwent surgery in long-term recorded follow-ups. Whereas, in our study most of the enrolled cases were mild to moderate degree cases, with short-term measured follow-ups. With this in mind, it is worth mentioning that steroid injections are believed to reduce perineural inflammation followed by reducing soft tissue swelling, and stabilizing the neural membrane [20], therefore our short-term study could better explain the corticosteroid effect of targeting inflammation in those cases.

Another key point to highlight is that it is known that nerves with axonal damage usually do not respond favorably to the use of a steroid. Therefore, it must be remembered that in this study severe CTS patients were often unresponsive to steroid injection as in the study of Meys et al. [14]. The most compelling evidence in our study, however, is that the participants with severe EMG degree did not appear to have weakness or atrophy on the thenar muscles, and consequently none of the participants had a carpal tunnel release operation during the recorded follow-ups. Therefore, we would emphasize that it could be postulated that there was no axonal damage on the median nerve in participants with severe EMG degree.

In 2009, Naranjo et al. [21] demonstrated that the CSA of a median nerve at the wrist is the only predictor of success after surgical release of transverse carpal ligament. In their study, patients with large maximal CSA of medi- 
an nerve at the wrist had a high success rate of a positive health outcome. In our study, patients with more swollen median nerve showed better outcomes as well. It is postulated that a severe inflammatory reaction can produce the result of making the median nerve more swollen, at which time the positive patient outcome responds better to the treatments as described.

In many previous studies [22-24], the CSA of median nerve at the wrist have been associated with the severity of CTS. In our study, although the sample size was small, the CSA of median nerve at the wrist tended to increase according to the severity of CTS (Supplementary Table S1). Despite the fact that the patients in this study revealed similar electrophysiological severity, patients with a larger CSA of median nerve showed better response to an application of a steroid injection. Thus, it is thought that in the short-term period, the reduction of inflammation might be the main factor of therapeutic effect after the application of a steroid injection.

In the analysis of changes in the measured NCS parameters after the procedure, the poor response group showed more likelihood of decrease of motor and sensory latency, and an increase of the sensory amplitude, than was noted in the good response group. Despite the fact that there were no statistical significances between the groups in theses parameters, these findings might indicate that there could be some clinical discrepancies between the subjective responses and electrophysiological findings. We think that these discrepancies were as a result of the number of participants having been statistically small in this case, and many patients with severe CTS were not enrolled in this study. In some previous studies [25,26], the clinical symptoms were significantly correlated with the electrophysiological severities of CTS. In contrast, Levine et al. [16] found an insignificant correlation between the overall symptom severity in CTS and conduction velocity of the median sensory nerve.

The BCTQ(SS) which was not different between groups before the procedure showed significant differences after the procedure. Namely, a decrease in pain by the procedure might explain this result. However, another key point was that the BCTQ(FS) showed the opposite result. It is noted that significant differences were observed before the procedure, but no further differences were shown or noteworthy after the procedure. Compared to the BCTQ(SS), BCTQ(FS) contains more specific item, such as 'writing activity' In our study, there were 5 patients who had bilateral symptoms and the other 20 patients had unilateral symptom on their dominant hands. This is thought to be the reason of vague results. Further study with many non-dominant hands should be followed.

It should be noted that this study had several specific limitations. First, we did not consider the possibility of influence regarding other factors, including the general health, patient mood, and physical examination findings as recorded for the patients. Second, there are well-known other sonographic factors that may have influenced the study, such as the wrist-to-forearm ratio (WFR), bowing of flexor retinaculum, flattening ratio, and mobility of median nerve. However, the CSA measurement is considered to be the simplest but most accurate measurement available for this study. Further study of this experiment should involve many sonographic variables, as mentioned above. Third, the follow-up period was relatively short and the total number of patients was small, but could be expanded in future studies. The further study with the utilization of long-term follow-up periods and larger number of patients should be followed to demonstrate the results of the current study.

In conclusion, the CSA of median nerve at wrist could be the strong predictive variable for the improvement of patient outcomes of the use of sonographically guided corticosteroid injection in mild-to-moderate CTS.

\section{CONFLICT OF INTEREST}

No potential conflict of interest relevant to this article was reported.

\section{SUPPLEMENTARY MATERIALS}

Supplementary materials can be found via https://doi. org/10.5535/arm.2018.42.2.213. Table S1. CSA of median nerve according to CTS severity.

\section{ACKNOWLEDGMENTS}

This work was supported by Electronics and Telecommunications Research Institute (ETRI) grand funded by the Korean government (No. 17ZR1400, Research on Beam Focusing Algorithm for Microwave Treatment). 


\section{REFERENCES}

1. Wong SM, Griffith JF, Hui AC, Tang A, Wong KS. Discriminatory sonographic criteria for the diagnosis of carpal tunnel syndrome. Arthritis Rheum 2002;46: 1914-21.

2. Buchberger W, Judmaier W, Birbamer G, Lener M, Schmidauer C. Carpal tunnel syndrome: diagnosis with high-resolution sonography. AJR Am J Roentgenol 1992;159:793-8.

3. Duncan I, Sullivan P, Lomas F. Sonography in the diagnosis of carpal tunnel syndrome. AJR Am J Roentgenol 1999;173:681-4.

4. Pastare D, Therimadasamy AK, Lee E, Wilder-Smith EP. Sonography versus nerve conduction studies in patients referred with a clinical diagnosis of carpal tunnel syndrome. J Clin Ultrasound 2009;37:389-93.

5. Uchiyama S, Itsubo T, Nakamura K, Kato H, Yasutomi T, Momose T. Current concepts of carpal tunnel syndrome: pathophysiology, treatment, and evaluation. J Orthop Sci 2010;15:1-13.

6. Hui AC, Wong S, Leung CH, Tong P, Mok V, Poon D, et al. A randomized controlled trial of surgery vs steroid injection for carpal tunnel syndrome. Neurology 2005; 64:2074-8.

7. Marshall SC, Tardif G, Ashworth NL. Local corticosteroid injection for carpal tunnel syndrome. London: The Cochrane Library; 2007.

8. Gelberman RH, Aronson D, Weisman MH. Carpaltunnel syndrome: results of a prospective trial of steroid injection and splinting. J Bone Joint Surg Am 1980;62:1181-4.

9. Kaplan SJ, Glickel SZ, Eaton RG. Predictive factors in the non-surgical treatment of carpal tunnel syndrome. J Hand Surg Br 1990;15:106-8.

10. Irwin LR, Beckett R, Suman RK. Steroid injection for carpal tunnel syndrome. J Hand Surg Br 1996;21:3557.

11. Weiss AP, Sachar K, Gendreau M. Conservative management of carpal tunnel syndrome: a reexamination of steroid injection and splinting. J Hand Surg Am 1994;19:410-5.

12. Agarwal V, Singh R, Sachdev A, Wiclaff, Shekhar S, Goel D. A prospective study of the long-term efficacy of local methyl prednisolone acetate injection in the management of mild carpal tunnel syndrome. Rheu- matology (Oxford) 2005;44:647-50.

13. Ollivere BJ, Logan K, Ellahee N, Miller-Jones JC, Wood M, Nairn DS. Severity scoring in carpal tunnel syndrome helps predict the value of conservative therapy. J Hand Surg Eur Vol 2009;34:511-5.

14. Meys V, Thissen S, Rozeman S, Beekman R. Prognostic factors in carpal tunnel syndrome treated with a corticosteroid injection. Muscle Nerve 2011;44:763-8.

15. Stevens JC. AAEM minimonograph \#26: the electrodiagnosis of carpal tunnel syndrome. American Association of Electrodiagnostic Medicine. Muscle Nerve 1997;20:1477-86.

16. Levine DW, Simmons BP, Koris MJ, Daltroy LH, Hohl GG, Fossel AH, et al. A self-administered questionnaire for the assessment of severity of symptoms and functional status in carpal tunnel syndrome. J Bone Joint Surg Am 1993;75:1585-92.

17. Delisa JA. Manual of nerve conduction velocity and clinical neurophysiology. Philadelphia: Lippincott Williams \& Wilkins; 1994.

18. Lee H, Kwon H. Electrophysiologic classification of severity of carpal tunnel syndrome. J Korean EMG Electrodiagn Med 2004;6:1-3.

19. Visser LH, Ngo Q, Groeneweg SJ, Brekelmans G. Long term effect of local corticosteroid injection for carpal tunnel syndrome: a relation with electrodiagnostic severity. Clin Neurophysiol 2012;123:838-41.

20. O'Gradaigh D, Merry P. Corticosteroid injection for the treatment of carpal tunnel syndrome. Ann Rheum Dis 2000;59:918-9.

21. Naranjo A, Ojeda S, Arana V, Baeta P, Fernandez-Palacios J, Garcia-Duque O, et al. Usefulness of clinical findings, nerve conduction studies and ultrasonography to predict response to surgical release in idiopathic carpal tunnel syndrome. Clin Exp Rheumatol 2009;27:786-93.

22. Lee CH, Kim TK, Yoon ES, Dhong ES. Correlation of high-resolution ultrasonographic findings with the clinical symptoms and electrodiagnostic data in carpal tunnel syndrome. Ann Plast Surg 2005;54:20-3.

23. Bayrak IK, Bayrak AO, Tilki HE, Nural MS, Sunter T. Ultrasonography in carpal tunnel syndrome: comparison with electrophysiological stage and motor unit number estimate. Muscle Nerve 2007;35:344-8.

24. Padua L, Pazzaglia C, Caliandro P, Granata G, Foschini M, Briani C, et al. Carpal tunnel syndrome: ultra- 
sound, neurophysiology, clinical and patient-oriented assessment. Clin Neurophysiol 2008;119:2064-9.

25. Leventoglu A, Kuruoglu R. Do electrophysiological findings differ according to the clinical severity of carpal tunnel syndrome? J Neurol Sci Turk 2006;23:272-8.
26. You H, Simmons Z, Freivalds A, Kothari MJ, Naidu $\mathrm{SH}$. Relationships between clinical symptom severity scales and nerve conduction measures in carpal tunnel syndrome. Muscle Nerve 1999;22:497-501. 


\section{SUPPLEMENTARY MATERIALS}

Table S1. CSA of median nerve according to CTS severity

\begin{tabular}{ccccc}
\hline & Mild (n=6) & Moderate $(\mathbf{n}=\mathbf{1 4})$ & Severe $(\mathbf{n}=\mathbf{5})$ & p-value \\
\hline $\mathrm{CSA}\left(\mathrm{mm}^{2}\right)$ & $12.1(9.8-12.8)$ & $14.1(11.9-15.9)$ & $18.6(17.2-18.9)$ & $0.004^{*}$ \\
\hline
\end{tabular}

Values are presented as median (interquartile range).

CSA, cross-sectional area; CTS, carpal tunnel syndrome.

${ }^{*} \mathrm{p}<0.05$ in Kruskal-Wallis analysis. 